\title{
THE EXTRAGALACTIC DIFFUSE BACKGROUND IN THE FAR ULTRAVIOLET
}

\author{
Francesco Paresce \\ Space Telescope Science Institute \\ 3700 San Martin Drive \\ Baltimore, MD 21218 USA \\ (Affiliated with Astrophysics Division, \\ Space Science Department, ESA)
}

\begin{abstract}
Due mainly to the minimal contaminating effects of zodiacal light and direct stellar emission, the far UV wavelength band from 912 to $\approx 2000 \AA$ is ideally suited, in principle at least, for an accurate measurement of a diffuse background component due to sources outside our own galaxy. The cosmological significance of such radiation is of great current interest as it certainly includes the cumulative line-of-sight effect of galaxies and quasars and may include emission from both a lukewarm intergalactic medium and decaying massive particles such as neutrinos, photinos, etc. The radiation required to maintain the IGM at its known high ionization level should also, in any case, appear clearly in this band due to redshift and lookback time effects, thereby providing a crucial clue as to its presently obscure origins. Just how accurately this component can be measured in practice, however, clearly depends on how well we understand the probably dominant galactic component from which it must be disentangled in one way or another except, possibly, at the galactic poles. The residual emission there in this band is on the order of a few $\times 10^{2}$ photons $\mathrm{cm}^{-2} \mathrm{~s}^{-1} \mathrm{sr}^{-1} \AA^{-1}$, of which perhaps as many as 50 units are almost certainly due to galaxies since the small-scale spatial fluctuations corresponding to this flux almost exactly mimic those expected from the known spatial distribution of galaxies. The rest must come from a presently uncertain source, most likely a residual tenuous dust layer at the galactic poles. This latter possibility is at least consistent with recent IRAS results on the diffuse IR background at $100 \mu \mathrm{m}$ and very sensitive $\mathrm{HI}, 21 \mathrm{~cm}$ measurements in these regions, but an extragalactic origin cannot be presently ruled out. Higher spatial and spectral resolution observations throughout the entire far UV range planned for the near future from orbiting platforms are expected to resolve this last but critically important issue.
\end{abstract}

\section{INTRODUCTION}

It has been recognized for some time now that:

(a) there is a detectable diffuse background radiation field at earth at all measurable wavelengths, and

(b) that it carries information that is of very significant cosmological interest.

The $2.7 \mathrm{~K}$ black body microwave background radiation that is the most straightforward signature of a hot big bang origin of the Universe is the most obvious, but certainly not the only, example that comes to mind. Similar situations exist in other wavelength bands from the gamma and X-ray to the IR. The optical region would seem the most suitable from an observational point of view as large collectors and great amounts of observing time can be directed towards the goal of accurately measuring even a very faint diffuse component. As Mattila has admirably shown (1989, this volume), however, there are a number of obstacles to the practical 
realization and satisfactory exploitation of this band for this purpose. Paramount among these is the contamination of the signal due to zodiacal light with smaller but still annoying contributions stemming from the effects of very faint stars and residual airglow emission.

Observations in the far UV band (912-2000 $\AA$ ) should, in principle, minimize or even completely eliminate most of these problems and, therefore, this band represents the ideal wavelength range for a thorough exploration of the galactic and extragalactic diffuse background, notwithstanding the difficulties associated with operating an instrument in space. These expectations are borne out in practice and for the following reasons. First, the zodiacal light intensity drops to zero below $\sim 2000 \AA$, due to the precipitous decline of the solar spectrum and the relative grayness of the interplanetary particle reflectance spectrum in the UV (see for example Röser and Staude, 1978). Second, the interstellar radiation field in the far UV is dominated by the contribution of $O$ and $B$ stars with $V$ magnitudes less than 10 that are either avoided altogether or easily identifiable and removable from the data base (Henry, 1977) and whose galactic latitude dependence is steep enough to ensure their rarity at $b \geqslant 50^{\circ}$ or so. Third, low altitude bright airglow emissions are entirely suppressed by the very high optical depth of far UV radiation in the atmosphere. The only remaining airglow lines of any significance in the far UV are the HI, Lyman $\alpha$ at $1216 \AA$ and the much weaker OI, 1304 and $1356 \AA$ features. Parenthetically, we should note that the necessity of avoiding even minimal sensitivity at these wavelengths has resulted in an unfortunate observational bias in favor of the region located above Lyman $\alpha$ to the detriment of the just as astronomically interesting but more technically challenging area just below it. The excellent data obtained recently from the Voyager spectrometer in this latter band (see Holberg, 1986; 1989, this volume) is finally pushing back even this last frontier.

In the following sections, a brief overview of the expected sources of the extragalactic background and their impact on astronomy will be presented, to be followed by a discussion of the biggest problem facing observers presently: the correct characterization and understanding of the galactic component. Recent results and observations bearing on this issue, especially some that are illustrative of the type of pitfalls occasionally encountered, will also be briefly described. Finally, the present status and future possibilities of this interesting endeavour will be addressed.

\section{EXPECTED EXTRAGALACTIC SOURCES OF FAR UV EMISSION}

\subsection{The Integrated Light of Galaxies and QSOs}

The line of sight integrated light of galaxies and quasars will give an average background $I_{\lambda}$ at wavelength $\lambda$ that is related to the redshift $z$, Hubble constant $H_{o}$ and the total density parameter $\Omega$ in a Friedman universe by the following expression (Paresce and Jakobsen, 1980):

$$
I_{\lambda}=\frac{c}{H_{o}} \frac{\varepsilon_{\lambda}}{4 \pi} \int_{o}^{\infty} \chi_{\lambda}[z, \lambda(1+z)] \phi(z)(1+z)^{-4}(1+\Omega z)^{-1 / 2} d z,
$$

where $\varepsilon_{\lambda}, \chi_{\lambda}(z, \lambda)$ and $\phi(z)$ are the luminosity density, mean spectrum and the comoving density evolution of the appropriate class of objects, respectively. The latter functions are normalized to $\chi_{\lambda}(o, \lambda)=1$ and $\phi(o)=1$. Absorption in the objects themselves is taken into account in $\chi_{\lambda}$ but intervening absorption between emitters is neglected.

Measurements of $I_{\lambda}$ can, in principle, constrain different galaxy and quasar evolution hypotheses. Under the assumption that the galaxies are opaque to radiation below the Lyman 
limit, one can probe the universe out to $z \approx 1.2$ with this technique. One can estimate the expected UV background from these sources using available information and educated guesses on their UV spectra and evolution. Results of these calculations are shown in Figure 1 adapted with some modifications from the review of Paresce and Jakobsen, 1980. The relevant observations and theoretical estimates of the contribution of a number of sources are given in this figure as a function of wavelength. The reader is referred to the original paper for a more detailed explanation of the calculations and meaning of the symbols.

If the color distribution of galaxies in the line of sight is such that it predominantly favors spiral galaxies, if the mean flux can be extrapolated to the Lyman limit with a power law, and if we neglect any evolution effects, the predicted contribution comes very close to the minimum observations (see curve marked Galaxies/2 in Figure 1). Thus, there is a distinct possibility that the integrated light of galaxies is presently being detected in some directions in the UV. On the other hand, it is more likely that the true situation will lie somewhere in between the two extremes (the curve marked Galaxies/1 in Figure 1 corresponds to a predominance of ellipticals and is therefore to be considered as a lower limit). As first pointed out by Tinsley (1972), $I_{\lambda}$ from equation 1 carries unique information on the evolution of star formation in galaxies. Indeed, using the observed lower limits of $\sim 300 C U(1 C U=1$ photon $\left.\mathrm{cm}^{-2} \mathrm{~s}^{-1} \mathrm{sr}^{-1} \AA^{-1}\right)$ to the observed $I_{\lambda}$, we can already place lower limits of $\sim 3 \times 10^{9}$ years or about a quarter of the Hubble time on the $e$-folding timescale for star formation leading to galactic luminosity evolution.

A probably better way to get at the extragalactic contribution due to galaxies is to look for small scale angular correlations or fluctuations of the measured intensity rather than just the average value given by equation (1). This is because we already know reasonably well from optical work the clustering properties of galaxies in the form of the spatial autocorrelation function given by Peebles, 1980. The expected radial power spectrum as a function of angular frequency is a power law with index -1.2 with a rather low amplitude corresponding to rms fluctuations $(\Delta / / I)$ of order $5 \%$ at $300 \mathrm{rad}^{-1}$ or $\sim 10^{\prime}$ angular scale (Martin and Bowyer, 1989). These fluctuations should not be too difficult to measure with present instrumentation and, indeed, probably have been recently observed (see Section 3).

\subsection{Radiative Recombination in a Lukewarm Photoionized IGM}

Although quasars seem to be, from the calculations shown in Figure 1, minor contributors to the background light, they play a crucial role in photoionizing a possible intergalactic medium (IGM) via their substantial light output below the Lyman limit at distant epochs. If the IGM is, in fact, highly ionized, the dominant emission mechanism would be radiative recombination in the H Ly $\alpha$ and HeII, $304 \AA$ lines. In this second case, the emitted intensity $I_{\lambda}$ per unit wavelength would be given by (Paresce, McKee and Bowyer, 1980):

$$
I_{\lambda}=\frac{\alpha_{l} x_{e} x_{j}\left[\bar{n}_{I G M}\left(z_{e m}\right) /\left(1+z_{e m}\right)^{3}\right]^{2} n c^{2} C e^{-<\tau} a^{(\lambda)>}}{2 H_{o}\left(1+\Omega z_{e m}\right)^{1 / 2} \lambda_{l}^{2}},
$$

where $\alpha_{l}$ is the effective recombination rate for the line, $x_{e}$ and $x_{j}$ are the ratios of free electrons and recombining ions, respectively, to baryons, and $\left\langle\tau_{a}(\lambda)\right\rangle$ is the mean absorption optical depth for He II, $304 \AA$ photons emitted at $1+z_{e m}=\lambda / \lambda_{l}$. The smeared out density of baryons in the IGM is given by:

$$
\bar{n}_{I G M}(z)=2.75 \cdot 10^{-6} h_{50}^{2} \Omega_{g}(1+z)^{3} \mathrm{~cm}^{-3},
$$




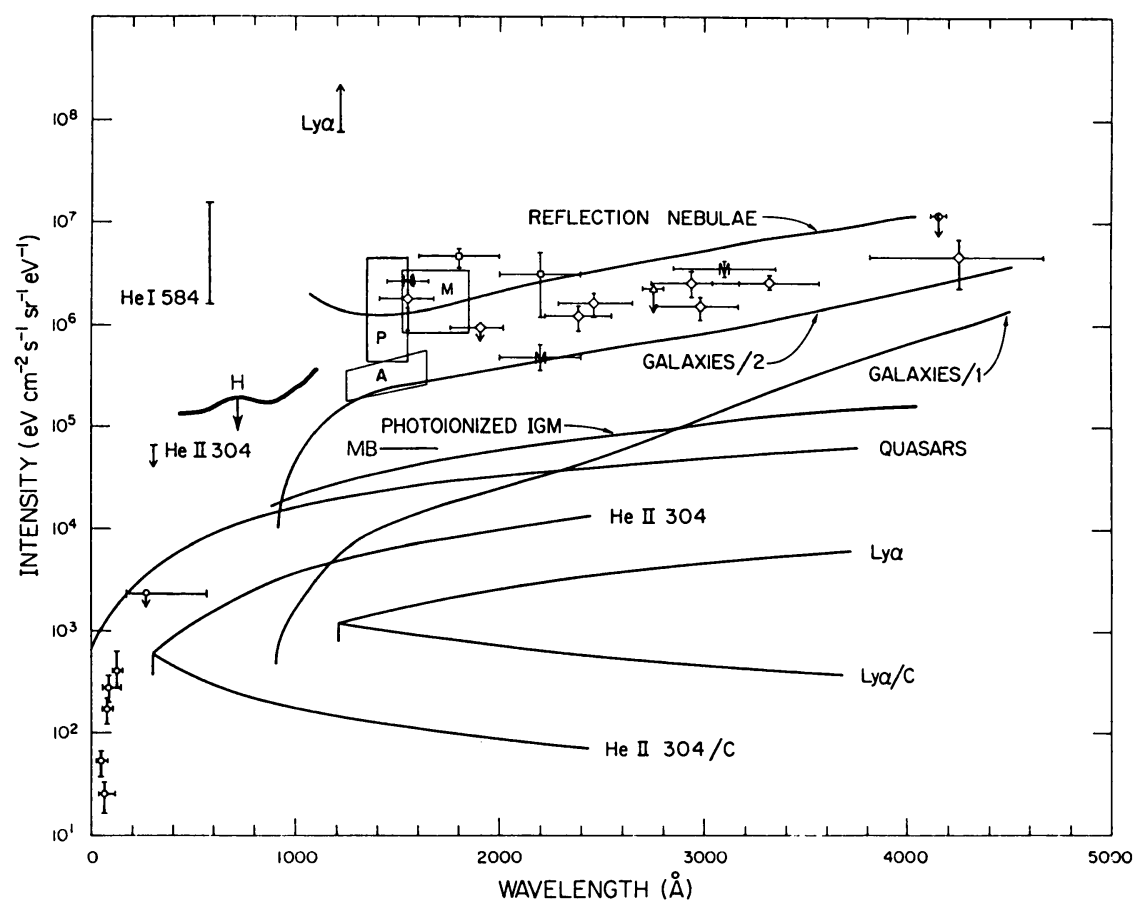

Figure 1. Observational and theoretical estimates of the diffuse UV background at high galactic latitudes as a function of wavelength. Sources of the observations: $A$, Anderson et al., 1979; point $H$, Henry et al., 1977; diamonds, Lillie and Witt, 1976; $M$, Maucherat-Joubert et al., 1979, 1980; triangle, Morgan et al., 1976; $P$, Paresce et al., 1979, 1980; circles, Paresce and Stern, 1981; squares, Pitz et al., 1979; filled circle, Spinrad and Stone, 1979; $M B$, Martin and Bowyer, 1989; curve $H$, Holberg, 1986.

where $h_{50}$ is the normalized Hubble constant $\left[h_{50}=H_{o} / 50 \mathrm{~km} \mathrm{~s}^{-1} \mathrm{Mpc}^{-1}\right]$ and $\Omega_{g}$ is the gas density parameter. Finally, the gas density distribution is taken into account by the clumping factor $C(z)=\left\langle n^{2}>\mid<n\right\rangle^{2}$. A number of predictions for a radiating highly ionized IGM with and without clumping at $T \sim 10^{4} \mathrm{~K}$ (curves marked Ly $\alpha$ and He II, 304) are shown in Figure 1 and in Figure 5 of Paresce, McKee and Bowyer, 1980. Most of these models lie several orders of magnitude below the lowest reported measurements. It is clear from these results that a substantial experimental effort will have to be mounted to penetrate down to these levels if the values of the input parameters are correct.

\subsection{Collisional Excitation in a Hot, Dense IGM}

One cannot completely rule out, however, the possibility that the IGM may have been reheated by dissipative processes some time in the past (Weymann, 1967; Sherman and Silk, 1979). In this case, collisional excitation of the HI Ly $\alpha$ and HeII, $304 \AA$ lines at the appropriate temperatures are the dominant emission processes. Because of the higher emissivity of collisionally excited plasma, this line emission, smeared by the redshift, could be significantly brighter than that due to recombination considered in equation (2). This scenario has been studied in detail by Jakobsen, 1980, who showed that under some very specific circumstances this source could contribute a good part, if not all, the observed radiation at $1400 \AA$. The observed 
background spectrum, the strict limits on neutral IGM hydrogen set by the Gunn-Petersen test, and the ad-hoc thermal histories required are serious obstacles to its wide acceptance, at least for the moment.

\subsection{The Source of IGM Ionization}

On the other hand, it is difficult to escape the conclusion that the high transparency of the IGM implied by the Gunn-Petersen test must be maintained by a high level of ionizing flux, a flux that should also turn up in the UV background. The curve "Photoionized IGM" in Figure 1, for example, is an estimate of the background level produced by redshifted $\mathrm{H}$ Ly $\alpha$ continuum radiation necessary to photoionize a dense IGM to the required level.

Unfortunately, no known sources, quasars included, seem capable of producing such a high flux, but there is mounting evidence that a substantial fraction of the mass of the universe is not baryonic and might have therefore gone undetected so far. This discrepancy is especially large if the universe is at the critical density as expected by current inflationary theories. If this unidentified material radiates in the EUV, it could well provide the required flux.

\subsection{Radiative Decay of Massive Particles}

The "missing" material may take the form of weakly interacting elementary particles of rest mass of tens of electron volts that may be produced in large quantities by pair production in the hot big bang. Several possibilities have been considered including massive neutrinos, photinos and gravitinos. A massive photino (the spin 1/2 partner of the photon) is predicted to have a near zero rest mass in the framework of spontaneously broken supersymmetry transformations while the gravitinos (the spin $3 / 2$ partner of the graviton) arise from supergravity theories.

Depending on precisely how and when they decouple from the thermal radiation field, neutrinos and/or photinos may dominate the mass of the universe, perhaps even providing the critical density. This cosmological sea of massive particles might be unstable to radiative decay. In the case of the neutrino, several decay channels are available, not all of them radiative (de Rujula and Glashow, 1980) but the decay of a heavy neutrino to a much lighter one plus a photon is preferred in many models. If the parent neutrino has a mass between 10 and $100 \mathrm{eV}$, the mean range most favored in present theoretical estimates and experiments, the corresponding decay photons have energies of 5 to $50 \mathrm{eV}$. The resultant emission beyond the rest wavelength of the decay photon $\lambda_{o}$ smeared by the redshift would have the form (Kimble, Bowyer and Jakobsen, 1981):

$$
I_{\lambda \geq \lambda_{o}}=\frac{c n_{\mathrm{v}}(z=0)}{H_{o} 4 \pi \tau} \frac{\lambda_{o}^{3 / 2}}{\lambda^{5 / 2}}\left[1+\left(2 q_{o}-1\right)\left(1-\lambda_{o} / \lambda\right)\right]^{-1 / 2} \exp (-t / \tau) .
$$

In this expression, $n_{\mathrm{v}}(z=0)$ and $\tau$ are the present volume density and lifetime of neutrinos, $t$ is the age of the universe at $z$ and $q_{o}$ the cosmological deceleration parameter. Kimble et al., 1981, have used observational upper limits to astronomical diffuse backgrounds to constrain $\tau \geq 10^{22}-20^{23} \mathrm{~s}$. In this case, the predicted emission peak from equation (4) is at the rest wavelength of the decay in the UV. Similar arguments can be advanced for the photino that may decay into a photon and a goldstino (Cabibbo et al., 1981).

Consequently, if there is a cosmological sea of elementary particles in quantities sufficient to close the universe, and provided these particles are unstable to radiative decay, it is quite likely that this huge diffuse mass could be detected by its characteristic emission in the UV. 
The emission would take the form of a continuum extended longwards of the rest wavelength $\lambda_{o}$ of the decay photon by the differential redshift associated with the expansion of the universe (see Figure 1 of Kimble et al., 1981). If these particles, moreover, provide the missing mass in our own galactic halo, we should also expect a monochromatic flux at the rest wavelength since the velocity dispersion of the galactic particles would be much smaller than $c$. In both cases, an unambiguous detection of this radiation would, obviously, be of fundamental importance both for cosmology and elementary particle physics.

\section{OBSERVATIONS}

As instructive examples of the use of far UV background data to extract potentially very useful information on the Universe, I have picked three recent episodes for the reader's consideration. All three make perfectly legitimate use of the currently available data. They also highlight some of the problems that can be encountered when overestimating the accuracy of the experimental results and/or relying too heavily on any specific theoretical model to the exclusion of other possibilities. The first example is the excellent fit obtained by Henry et al., 1978, using their data and Weymann's (1967) model for collisional excitation in a dense intergalactic plasma model (see Section 2.3). Consistency between this theory and data set is achieved for a value of $H_{o}=64$, thereby yielding a new independent determination of this crucial parameter and resolving the well known ambiguity inherent in other less accurate determinations.

The second intriguing case of the use of far UV data is reported by Maalampi, Mursula and Roos, 1986, whereby a purported step in the background spectrum of $\approx 500 \mathrm{CU}$ size at $\approx 1670 \AA$ is interpreted as evidence of the decay of neutrinos of mass $14.9 \mathrm{eV}$ with a lifetime of order of $10^{16}$ years. The latter is, of course, many orders of magnitude lower than expected from the standard electroweak interaction theory that assumes a purely left-handed structure of the charged weak currents. Thus, this result is convincingly used by the cited authors to imply that the standard model must be modified to also include right-handed currents. In one swoop the existence of a radiatively decaying neutrino, its mass and the need for a new, modified standard theory is legitimately established. Unfortunately, it is not so clear whether such a step in the spectrum actually exists. Present consensus among observers seems to be negative on this point, but a healthy and open mind needs to be a constant attribute in this field as such a spectrum certainly cannot be completely ruled out at the moment.

A third example of a perhaps more conservative exploitation of the far UV background is that reported by Martin and Bowyer (1989). Their approach follows that first suggested by Shectman (1973) by adding to the average intensity information the one derived from the measured amplitude and angular frequency dependence of the fluctuation in the background intensity. Using this method, the cited authors find solid evidence for an extragalactic component due to the integrated far UV light of galaxies corresponding to $\approx 10-20 \%$ of the total average intensity, i.e., $30-50 \mathrm{CU}$ in the $1350-1900 \AA$ band. This result is marked MB in Figure 1 and, as predicted in Section 2.1, is comfortably bracketed by the two curves marked Galaxies/1 and 2. This important result is taken by MB to imply that the mean galaxy luminosity out to $z=0.6$ could not be more than $50 \%$ greater than at present and, provided no changes in absorption or in the initial mass function have occurred, that the mean star formation rate in the Universe has not changed significantly in this same time interval. 


\section{THE GALACTIC BACKGROUND}

A discussion of the extragalactic background cannot be complete without careful consideration of the foreground galactic background which is, most probably, everywhere significantly brighter than the former even at the galactic poles. It has become recently obvious that unless this dominant component is well understood and completely characterised, little progress can be expected on the extragalactic front beyond the present situation. This situation is rather exhaustively summarized by Paresce and Jakobsen (1980), Paresce (1985), Martin and Bowyer (1989) and Lequeux (1989, this volume). Much progress has been made on this front in the last few years after the initial confusion following the discovery of the relationship between $I_{\lambda}$ and N(HI) reported by Paresce, McKee and Bowyer (1980).

At this point in time we are reasonably confident of the following:

(a) The galactic component of the far UV background has an average intensity that is quite variable from place to place in the sky with values ranging from a minimum of $300 \pm 100 \mathrm{CU}$ to a maximum of $1-2 \times 10^{3} \mathrm{CU}$.

(b) This component is definitely related to the line of sight $\mathrm{N}(\mathrm{HI})$ obtained from $21 \mathrm{~cm}$. radio observations (see Lequeux, 1989, this volume, for the latest and best survey data on this point).

(c) Both the observed average intensity and its general correlation with $\mathrm{N}(\mathrm{HI})$ is well reproduced and understood in terms of a simple dust scattering model in which dust clouds above the galactic plane scatter far UV radiation emitted by hot young stars in the plane back to the observer (see curve marked Reflection Nebulae in Figure 1).

(d) The average spectrum of the far UV background is slowly varying with wavelength with a possible drop below $1200 \AA$ (Holberg, 1986) and a possible increase above $\approx 1700 \AA$.

These results find confirmation and support in the optical range where the "infrared cirrus" was discovered long before IRAS (Sandage, 1976) even at high galactic latitudes and, especially, in the far IR at $100 \mu$ where the scattering grains become directly visible and their correlation with N(HI) clearly established (Boulanger and Perault, 1988; Boulanger, 1989, this volume). Moreover, since we do know from true reflection nebulae surrounding luminous stars that the grain albedo in the UV is definitely $>0$, at this point we would have real trouble explaining why we would not see the dust in the far UV via this mechanism.

All this does not mean, however, that all is understood. There are a number of intriguing and unanswered questions that still need addressing. Among others they are:

(a) Why is the $I$ vs. $\mathrm{N}(\mathrm{HI})$ relationship as ragged as it appears? Is it due to variations in illumination, in grain properties, in optical depth effects, or a combination of all these?

(b) Why is the $I_{\lambda}(1200-2000 \AA)$ vs. $I_{\lambda}(100 \mu)$ as poor as it is (see Jakobsen, deVries and Paresce, 1987)?

(c) Are other sources of emission present such as $\mathrm{H}_{2}$ or $\mathrm{CO}$ fluorescence, hot gas line emission, two photon continuum from the ionized gas, etc., and what is their relative weight in any direction?

(d) What is the source(s) of the excess UV emission seen in many areas of the sky (see Paresce, Jakobsen and Bowyer, 1983 for example)? 
(e) What is the significance of the "apparent" residual intensity at $\mathrm{N}(\mathrm{HI})=0$ of the far $\mathrm{UV}$ background at $300 \pm 100 \mathrm{CU}$ discussed earlier?

All these questions clearly need an answer which, when it comes, will certainly yield significantly more insight into the structure and behaviour of our galaxy. The last question, on the other hand, has a more direct impact on the topic of this review and a first attempt at answering it was made in an earlier paper (Paresce, 1985). The situation has not changed too dramatically since that time, but we do now know thanks to the work of Lockman, Jakoda and McCammon, 1986 that a real $\mathrm{N}(\mathrm{HI})=0$ line of sight may not really exist anywhere in the galaxy. This fact prompts my use of the term "apparent" in question (e) above. In fact, using equation (6) from Paresce (1985) to compute the expected $I_{\lambda}$ from the data of Lockman et al. of $\mathrm{N}(\mathrm{HI})=4.5 \cdot 10^{19} \mathrm{~cm}^{-2}$ which uses the canonical values for the free parameters, one obtains $I_{\lambda} \approx 50 \mathrm{CU}$, which is small but far from negligible, especially considering the significant error associated with it.

It is instructive in this context to study the spatial distribution of IR-emitting grains at the polar caps to be found in Figure 7 of Boulanger and Perault (1988). Again, assuming the average relationship between $\mathrm{N}(\mathrm{HI}), I_{\lambda}$ (far UV) and $I_{\lambda}$ (far IR) holds everywhere, we can transform these IR maps into approximate far UV maps. In this scenario, the white areas correspond to $I_{\lambda}$ (far UV) < $100 \mathrm{CU}$ ("Lockman's hole" falls nicely in one of these) and the first gray level to the range 113-265 CU, the second level to 265-380 CU and the third or darkest to 380-570 CU to cover most of the observed intensity range in the far UV at least near the poles. Although the scenario is certainly oversimplified, it should represent rather faithfully the rough outlines of the expected far UV radiation pattern. But what about the extragalactic component? Is there any direction in which this component is free from galactic contamination?

The rough coincidence between my computed expectation of $I_{\lambda}$ (far UV) in "Lockman's hole" and Martin and Bowyer's measurement described in Section 3 would seem at least promising in this regard. Whether or not this is a fortuitous coincidence really depends on the origin of the residual background of magnitude $1.2-1.8 \mathrm{MJy} \mathrm{sr}^{-1}$ at $100 \mu \mathrm{m}$ discussed in some detail by Boulanger and Pérault (1988) and by Boulanger (1989, this volume). This flux could all be of extragalactic or interplanetary origin, of course, or it could be due to a thin veil of dust that covers most, if not all, the solar neighbourhood. In the latter case, we could not expect to find regions of far UV intensity much lower than the few $\times 10^{2} \mathrm{CU}$ just mentioned, making the direct measurement of an extragalactic component much more difficult.

\section{CONCLUSIONS}

In our rather mad, head-long rush to the "Holy Grail" we have tended in our impatience to brush aside some very important, exciting, and intriguing issues referring mainly to our own galaxy rather than the IGM (possibly mythical anyway). We have now realized, however, that these questions are not only just as scientifically rewarding in their own right, but that very little progress to the Holy Grail will ever be made unless we stop to understand our own more prosaic backyard first. Once that is accomplished, the rewards will surely be great, since quite a few known (see Figure 1) and, possibly, presently unknown extragalactic sources are tantalizingly close to the region of present observability (10-100 CU). Of special significance in this endeavour will be the correct understanding of the origin of the residual flux both in the far UV and IR at high galactic latitudes that is currently mysterious. Of this residual, we have 
established the important fact that only about $10-20$ is due to the integrated light of galaxies, the rest being unaccounted for. It could be residual dust, two photon emission, molecular fluorescence, etc.

The observational situation in the far UV remains uncertain even though there is certainly no lack of excellent ideas (see papers in Section V of this volume). Our hopes for the near future thus rest on the next shuttle flight carrying the FAUST camera and on further analysis of D2B and UVX data. Unfortunately, a really powerful, comprehensive all-sky spectroscopic survey is further away into the future than we would like (see the HUBE and PAX proposed missions described by Kimble, Henry, and Paresce, and Martin and Steiner, respectively; both 1989, this volume). We can only hope that these important endeavours will be funded in the near future.

I sincerely hope that the results of this symposium will go far in aiding such a just cause in showing the truly profound information that can be gleaned from such investigations.

\section{REFERENCES}

Anderson, R. C., Brune, W. H., Henry, R. C., Feldman, P. D., and Fastie, W. G. 1979, Ap. J. (Letters), 233, L39.

Boulanger, F. 1989, in Proc. IAU 139, Galactic and Extragalactic Background Radiation, ed. S. Bowyer and Ch. Leinert, Dordrecht, Kluwer Academic Publishers.

Boulanger, F., and Perault, M. 1988, Ap. J., 330, 964.

Cabibbo, N., Farrar, F. R., and Maiani, L. 1981, Phys. Rev. Lett. B, 105, 155.

De Rujula, A., and Glashow, S. 1980, Phys. Rev. Lett., 45, 942.

Henry, R. C. 1977, Ap. J. Suppl., 33, 451.

Henry, R. C., Feldman, P. D., Fastie, W. G., and Weinstein, A. 1978, Ap. J., 223, 437.

Henry, R. C., Swandic, J. R., Shulman, S. D., and Fritz, G. 1977, Ap. J., 212, 707.

Holberg, J. B. 1986, Ap. J., 311, 969.

Holberg, J. B. 1989, in Proc. IAU 139, Galactic and Exgragalactic Background Radiation, ed. S. Bowyer and Ch. Leinert, Dordrecht, Kluwer Academic Publishers.

Jakobsen, P. 1980, Astr. Ap., 81, 66.

Jakobsen, P., de Vries, J., and Paresce, F. 1987, Astr. Ap., 183, 335.

Kimble, R. A., Bowyer, S., and Jakobsen, P. 1981, Phys. Rev. Lett., 46, 80.

Kimble, R. A., Henry, R. C., and Paresce, F. 1989, in Proc. IAU 139, Galactic and Extragalactic Background Radiation, ed. S. Bowyer and Ch. Leinert, Dordrecht, Kluwer Academic Publishers.

Lequeux, J. 1989, in Proc. IAU 139, Galactic and Extragalactic Background Radiation, ed. S. Bowyer and Ch. Leinert, Dordrecht, Kluwer Academic Publishers.

Lillie, C. F., and Witt, A. N. 1976, Ap. J., 208, 64.

Lockman, F., Jakoda, K., and McCammon, D. 1986, Ap. J., 302, 432.

Maalampi, J., Mursula, K., and Roos, M. 1986, Phys, Rev. Lett., 56, 1031.

Martin, C., and Bowyer, S. 1989, Ap. J., 338, 677.

Martin, C., and Steiner, J. 1989, in Proc. IAU 139, Galactic and Extragalactic Background Radiation, ed. S. Bowyer and Ch. Leinert, Dordrecht, Kluwer Academic Publishers.

Mattila, K. 1989, in Proc. IAU 139, Galactic and Extragalactic Background Radiation, ed. S. Bowyer and Ch. Leinert, Dordrecht, Kluwer Academic Publishers.

Maucherat-Joubert, M., Cruvellier, P., and Deharveng, J. M. 1979, Astr. Ap., 70, 467.

Maucherat-Joubert, M, Deharveng, J. M, and Cruvellier, P. 1980, Astr. Ap., 88, 323.

Morgan, D. H., Nandy, K., and Thompson, G. I. 1976, M.N.R.A.S., 117, 531.

Paresce, F. 1985, Il Nuovo Cimento, 8C, 379.

Paresce, F., and Jakobsen, P. 1980, Nature, 288, 119.

Paresce, F., Jakobsen, P., and Bowyer, S. 1983, Astr. Ap., 124, 300.

Paresce, F. Margon, B., Bowyer, S., and Lampton, M. 1979, Ap. J., 230, 304.

Paresce, F., McKee, C., and Bowyer, S. 1980, Ap. J., 240, 387.

Paresce, F., and Stern, R. 1981, Ap. J., 247, 89.

Peebles, P. J. E. 1980, The Large Scale Structure of the Universe, Princeton Univ. Press. 
Pitz, E., Leinert, C., Schultz, A., and Link, H. 1979, Astr. Ap., 72, 92.

Röser, S., and Staude, H. J. 1978, Astr. Ap., 67, 381.

Shectman, S. A. 1973, Ap. J., 179, 681.

Sandage, A. 1976, A. J., 81, 954.

Sherman, R. D., and Silk, J. 1979, Ap. J. Letters, 231, L61.

Spinrad, H., and Stone, R. P. S. 1979, Ap. J., 226, 609.

Tinsley, B. M. 1972, NASA SP-310, 375.

Weymann, R. 1967, Ap. J., 147, 887.

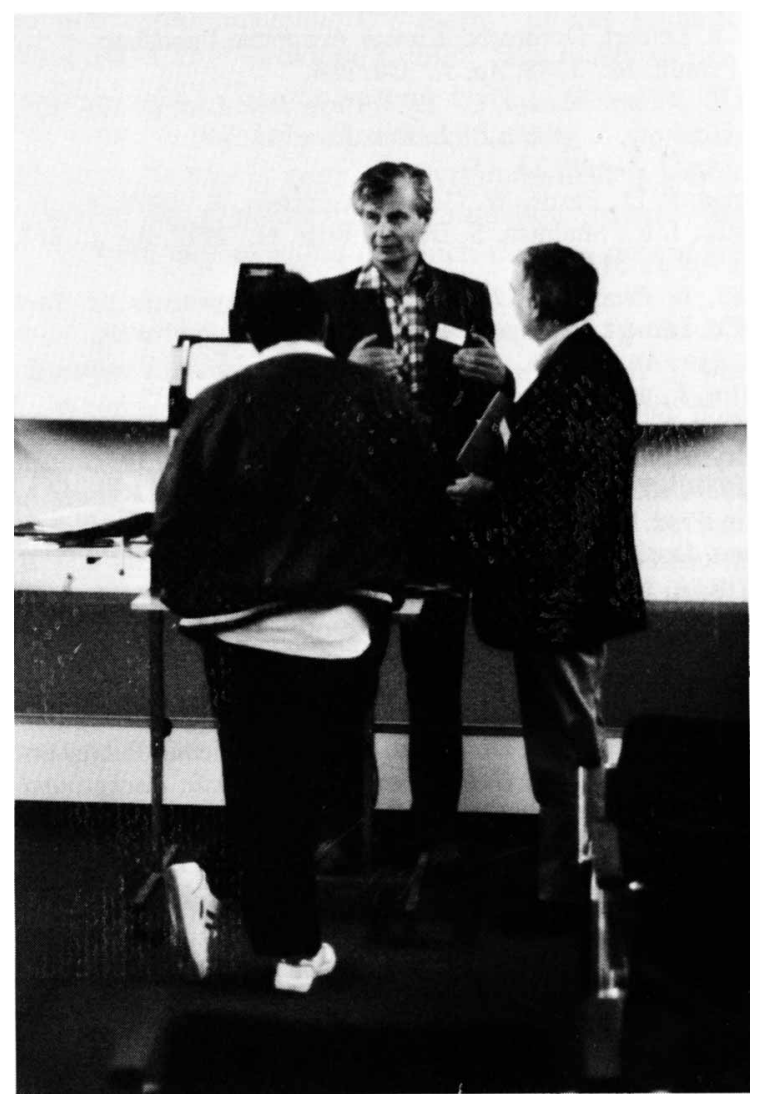

I.A. Strukov 